\title{
Evaluación de una modificación en la norma ASTM C1609 para el estudio de concreto reforzado con fibras sintéticas sometido a flexión
}

Evaluation of a modification in ASTM C1609 standard for the study of synthetic fiber reinforced concrete under flexural load

\section{Kenneth J. Paniagua Murillo}

Estudiante, Escuela de Ciencia e Ingeniería de los Materiales Instituto Tecnológico de Costa Rica, Cartago, Costa Rica paniaguakenneth717@gmail.com

\section{M.Sc. Ing. Francisco Villalobos Ramírez}

Jefe Laboratorio de Estructuras, Lanamme-UCR

Universidad de Costa Rica, San José, Costa Rica

francisco.villalobosramirez@ucr.ac.cr
Índices y Bases de Datos:

lationdex ucrindex
- Dialnet
DOA
RIÓDICA

REDIB

\section{Ulbiblat ERIHPDUS}

Políticas de Uso:

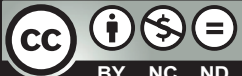

Revista Métodos y Materiales por LanammeUCR se distribuye bajo: Licencia Creative Commons Atribución-NoComercialSinDerivar 4.0 Internacional. ISSN electrónico: 2215-4558 


\title{
Evaluación de una modificación en la norma ASTM C1609 para el estudio de concreto reforzado con fibras sintéticas sometido a flexión
}

\author{
Evaluation of a modification in ASTM C1609 standard for the study of synthetic fiber reinforced \\ concrete under flexural load
}

\section{Kenneth J. Paniagua Murillo}

Estudiante, Escuela de Ciencia e Ingeniería de los Materiales Instituto Tecnológico de Costa Rica, Cartago, Costa Rica

paniaguakenneth717@gmail.com
M.Sc. Ing. Francisco Villalobos Ramírez

Jefe Laboratorio de Estructuras, Lanamme-UCR

Universidad de Costa Rica, San José, Costa Rica

francisco.villalobosramirez@ucr.ac.cr

Fecha de recepción: 7 agosto 2019 / Fecha de aprobación: 4 marzo 2020

\section{RESUMEN}

En esta investigación se evaluó la viabilidad de una modificación a la norma ASTM C1609 con el fin de determinar la conveniencia en la aplicación, o no, de ese estándar para ensayar concreto reforzado con fibras sintéticas (SNFRC) sometido a flexión. También se realiza una comparación entre los resultados de tal norma con respecto a los que provee el estándar europeo EN14651. Se determinó un comportamiento pseudodúctil del material en el que la incorporación de fibras le permite resistir cierta carga aun después de alcanzar su módulo de ruptura. La reducción en la velocidad de ensayo aumentó las probabilidades de tener resultados exitosos vía ASTM C1609, especialmente cuando el SNFRC tiene poca fibra añadida. Además, la modificación de velocidad no implicó alteraciones en los resultados del ensayo. Por otra parte, se encontró que la norma europea no produce problemas en la ejecución del ensayo, sin embargo, sus resultados no fueron en todos los casos comparables con lo obtenido a través de la norma norteamericana.

Palabras clave: capacidad residual, concreto reforzado con fibras sintéticas, módulo de ruptura, tasa de aplicación de carga.

\begin{abstract}
In this research, it was evaluated the viability of modification in the procedure established in ASTM C1609 in order to determine if it is applicable, or not, for testing of synthetic fiber reinforced concrete (SNFRC). A comparison between ASTM $\mathrm{C} 1609$ and EN14651 results was realized. A pseudoductil behavior was found for SNFRC where the addition of fibers allowed concrete to resist loads after its modulus of rupture is reached. The reduction in loading rate taste established in ASTM C1609 give higher probability of success for the test, mainly when low fiber content is used. The results were not affected by the variation in loading rate taste. Finally, the European standard did not cause any inconvenience in the test execution; however, its results were not comparable with the American standard results.
\end{abstract}

Keywords: load application rate, modulus of rupture, residual capacity, synthetic fiber reinforced concrete. 


\section{INTRODUCCIÓN}

Desde tiempos antiguos se utilizan fibras para reforzar materiales frágiles, por ejemplo, se empleaba la paja para reforzar ladrillos. Actualmente diversos materiales ingenieriles incorporan fibras con el objetivo de crear compuestos con propiedades mejoradas (ACI Comittee 544, 1996). El concreto reforzado con fibra sintética es más fácil de fabricar que aquel reforzado con varillas de acero, pues las fibras solo se distribuyen en la mezcla y además representan costos de producción menores (ACI Comittee 544, 1996).

\section{Concreto reforzado con fibras sintéticas (SNFRC)}

El SNFRC consiste en una matriz cerámica constituida por cemento hidráulico y agregados a la que se le incorporan fibras cortas y discontinuas dispersas (ACI Comittee 544, 1996; Zongjin, 2017); esta clase de FRC se clasifica como FRC Tipo III (ASTM International, 2009).

El comportamiento del material compuesto concreto-fibra está determinado por las propiedades físicas de la matriz y de las fibras, así como por la fuerza de interacción que existe entre ambas, la unión es meramente mecánica (ACI Comittee 544, 1996; Zongjin, 2017).

Los materiales frágiles sometidos a esfuerzo inicialmente se deforman elásticamente y pierden cualquier ductilidad después de la formación de la primera grieta, sin embargo, la adición de fibras al concreto cambia ese comportamiento: las fibras no aumentan el esfuerzo necesario para la formación de la primera grieta pero sí mejoran la respuesta post-agrietamiento, estos aspectos se evalúan a partir de la tenacidad del material (ACI Comittee 544, 1996).

Al formarse la primera grieta, la resistencia del concreto ordinario cae rápidamente, pero en el SNFRC las fibras actúan como puentes entre las grietas de modo que ocurre la transferencia de cargas. La reducción gradual de la carga con la elongación en el SNFRC es debida a la extracción de las fibras del interior de la matriz cuando se alcanza un valor crítico de esfuerzo cortante en su interfaz con el concreto; si el polímero falla de manera rápida, no hay transferencia de carga y el modo de falla será el de concreto frágil común (P. Kumar Mehta \& Paulo J. M. Monteiro, 2014; Zongjin, 2017). En congruencia, la teoría de líneas de fluencia de Meyerhoff indica que la adición de fibras permite la redistribución de esfuerzos en la sección del concreto de modo que existe una capacidad residual después del agrietamiento (Navas Carro \& Rojas Juárez, 2010).

Un problema del uso de fibras como refuerzo es que su distribución en la matriz, su posición con respecto a la dirección de formación de grietas y su longitud de empotramiento con la matriz pueden ser deficientes y, por tanto, es posible que solo un pequeño porcentaje del total de fibra agregado esté beneficiando la resistencia tensión y a flexión de un concreto (Kosmatka, Kerkhoff, \& Panaresse, 2002). Además, las fibras reducen la trabajabilidad del concreto, sin embargo, esto no suele ser un problema muy grave cuando la compactación del mismo se hace por vibración (ASTM International, 2009).

\section{Comparación entre las normas ASTM C1609 y EN14651}

La norma ASTM C1609 permite obtener la curva de carga contra deflexión neta de una viga hasta que se alcance la deflexión de 1/150 veces la longitud que existe entre los puntos de apoyo; la tasa de incremento de la deflexión es dependiente del valor de deflexión medido. El ensayo es tipo flexión en cuatro puntos (ASTM International, 2012). La aplicación de la carga desde dos puntos implica que entre ellos se genera una región de flexión pura en la viga, si aparecen grietas fuera de esa región el ensayo debe ser descartado (Paegle \& Fischer, 2014).

Por otra parte, en el ensayo según EN14651 la viga es soportada en dos puntos y sometida a una carga en su punto medio. Se grafica la relación entre la apertura o CMOD. El ensayo se ejecuta hasta que el CMOD sea al menos de 4 $\mathrm{mm}$ y se rechaza cualquier viga cuyas grietas se empiecen a formar fuera de la muesca. Los valores de CMOD se pueden transformar a deflexión neta (European Comittee for Standardization, 2005). En realidad, el transductor no mide todas las deformaciones que ocurren, pues este solo registra el CMOD y no lo que ocurre en el resto de la viga (Paegle \& Fischer, 2014).

Ambas normas permiten calcular la capacidad residual del material; en el caso de ASTM C1609 el valor se denomina y la norma da la forma de obtenerlo mientras que en EN14651 se debe calcular como según el reporte TR-63 de la Sociedad del Concreto. Son parámetros equivalentes (ASTM International, 2012). 
Al emplear la norma ASTM C1609 se ha visto que ocurre una falla súbita de las probetas, no es posible apreciar su comportamiento residual. Según estudios previos (Banthia \& Islam, 2013; Valenzuela, 2010), la velocidad de ensayo es el parámetro por el cual las probetas se fracturan rápidamente. Por tanto, este trabajo tiene por objetivo evaluar el efecto de una modificación en las velocidades de ensayo dadas en la norma ASTM C1609 para lograr estudiar el comportamiento en flexión del SNFRC sin que se presente su falla súbita, así como comparar los resultados arrojados por esta norma respecto a los obtenidos según EN14651.

\section{MATERIALES Y MÉTODOS}

\section{Caracterización de los agregados y cemento}

El agregado fino corresponde a arena de río y el grueso a grava, para ambos se determinó su contenido de humedad, gravedad específica y absorción, peso unitario y se realizó su granulometría; los ensayos fueron de acuerdo a las normas ASTM C566-13, C127-15/C128-15, C29-17a y C136-14, respectivamente. Se utilizó cemento tipo industrial MC/AAR, cuya gravedad específica se encontró por ASTM C188-17.

\section{Preparación de mezclas de concreto de prueba}

Las mezclas de prueba se prepararon de acuerdo con ASTM C192-18. Para identificar los cilindros preparados en cada mezcla de prueba se usó el siguiente código: $\mathbf{C 1 0 3}$. Donde la C se refiere a cilindro, el primer número es el número de mezcla de prueba y los últimos dos corresponden a la cantidad de días después de la colada en que se realizó el ensayo de compresión dado en ASTM C39-18 y con el equipo Humboldt, activo UCR279217.

\section{Fabricación de las vigas de SNFRC}

La fibra polimérica utilizada fue la Ruredil XFiber 54, aportada por la empresa SaHe S.A.; la fibra es suministrada como una mezcla de los filamentos mostrados en la Figura 1. Consiste en una mezcla de monofilamentos poliolefínicos no fibrilados y fibra fibrilada de polipropileno.

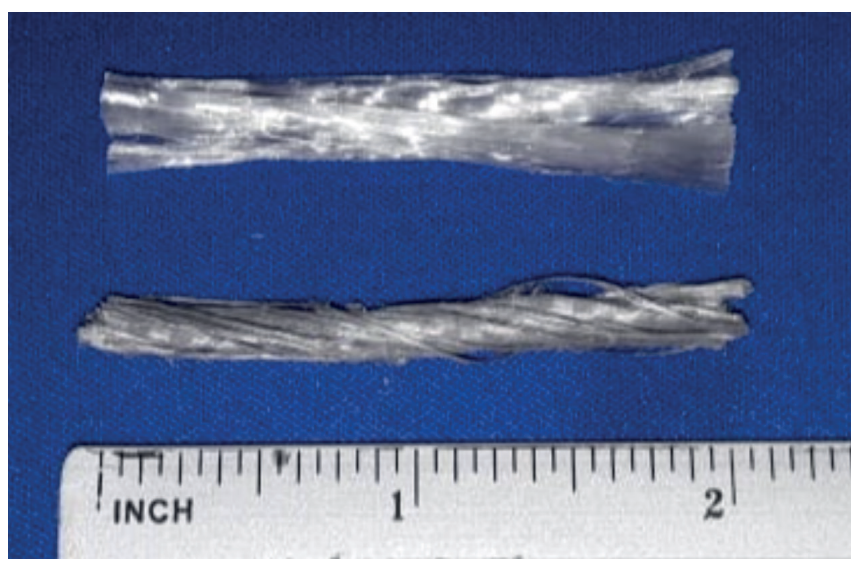

Figura 1. Fibra Ruredil XFiber 54 utilizada para elaborar el SNFRC.

Se fabricaron 24 vigas, 12 con dosificación de $1,5 \mathrm{~kg} / \mathrm{m}^{3}$ de fibra y las restantes con $5,5 \mathrm{~kg} / \mathrm{m}^{3}$. El moldeo se hizo en grupos de 4, además se colaron dos cilindros con diámetro de $10 \mathrm{~cm}$ y altura de $20 \mathrm{~cm}$ para el control de la resistencia a compresión de la mezcla producida cada día de colada. La preparación de las vigas también se llevó a cabo según ASTM C192, únicamente fue necesario suspender el curado por un tiempo corto las vigas que se fallarían según EN14651 para generarles la muesca. En las coladas que se estimó necesario, se incorporó el aditivo plastificante Ruredil Ergomix 6005. La compactación del SNFRC se hizo por vibración externa con una mesa Syntron VP-51-D1, activo UCR376654.

Para la identificación de cada viga se empleó el siguiente código: BEN1, donde la primera letra se refiere a la dosis baja (B) o alta (A), la última indica el número de probeta ensayada y las letras intermedias se refieren a la norma utilizada para el ensayo en flexión: "EN" para EN14651, "AS" para ASTM C1609 y "ASM" para ASTM C1609 con la modificación planteada. Los cilindros fabricados junto a los grupos de vigas se identifican como: CBAS. La primera letra se refiere al tipo de sección transversal, la segunda a la dosis de fibra, las siguientes letras indican con cuál grupo de cuatro vigas se fabricó el cilindro: "AS" junto a vigas ensayadas vía ASTM C1609, "ASM" con la misma norma, pero modificada y "EN" si es con la europea. 


\section{Ensayos De Flexión}

Un primer grupo de 4 vigas se ensayó por ASTM C1609, el segundo de acuerdo a la versión modificada de la misma norma y el grupo restante según EN14651. En la Figura 2 está mostrada la configuración de los ensayos, todos se realizaron en el equipo MTS810, máquina universal con capacidad de

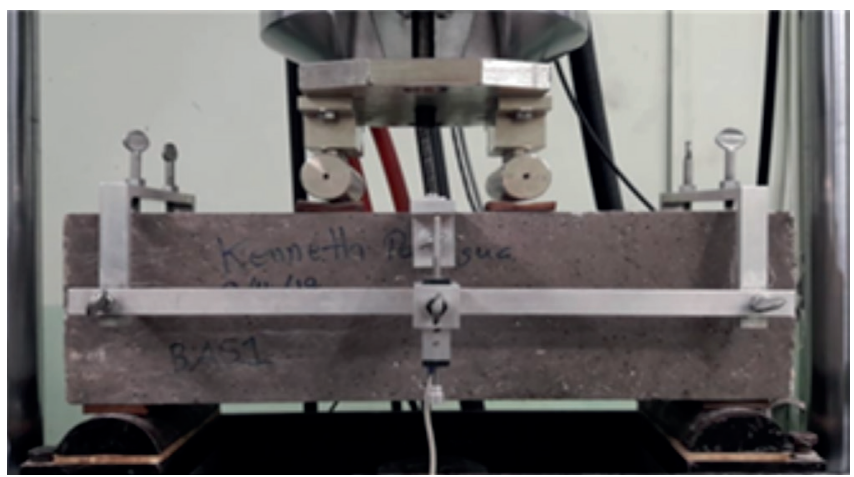

(a)
250 kN, UCR218602. Las tasas de aplicación de carga para la norma ASTM C1609 estándar y modificada se muestran en la Tabla 1. Para EN14651 fue de $0,06 \mathrm{~mm} / \mathrm{min}$ hasta un CMOD de $0,124 \mathrm{~mm}$, de ahí en adelante se utilizaron $0,2 \mathrm{~mm} / \mathrm{min}$.

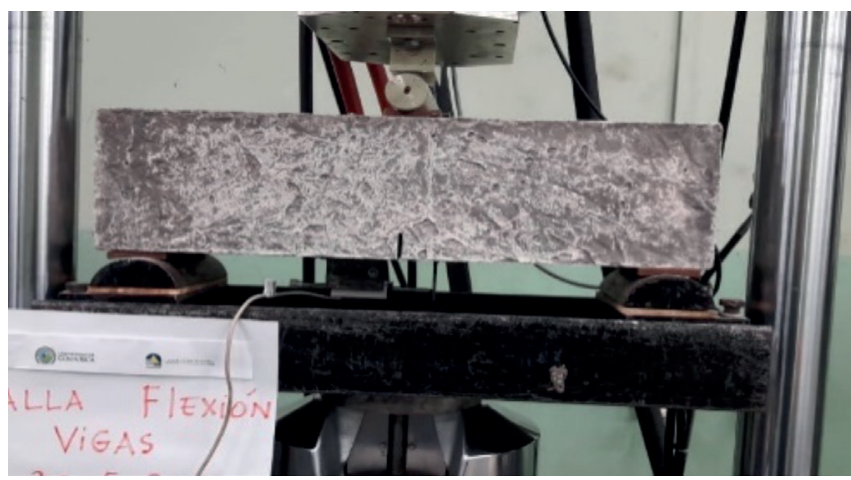

(b)

Figura 2. Configuración del ensayo de flexión para a) ASTM C1609 y b) para EN14651.

Tabla 1. Velocidades de ensayo para ensayos con la norma ASTM C1609 estandar y modificada.

\begin{tabular}{|c|c|c|c|c|}
\hline \multicolumn{2}{|c|}{ Velocidad de ensayo } & \multirow{2}{*}{$\begin{array}{l}\text { Desplazamiento } \\
\text { vertical (mm) }\end{array}$} & \multirow{2}{*}{$\begin{array}{l}\text { Desplazamiento } \\
\text { vertical neto }(\mathrm{mm})\end{array}$} & \multirow[b]{2}{*}{ Norma } \\
\hline Incremento & $\begin{array}{l}\text { Tasa de deflexión } \\
(\mathrm{mm} / \mathrm{min})\end{array}$ & & & \\
\hline Inicial & 0,035 & 0,500 & 0,500 & \multirow{5}{*}{ ASTM C1609 } \\
\hline Primero & 0,085 & 0,325 & 0,825 & \\
\hline Segundo & 0,135 & 0,375 & 1,200 & \\
\hline Tercero & 0,185 & 0,425 & 1,625 & \\
\hline Cuarto & 0,235 & 1,625 & 3,250 & \\
\hline Inicial & 0,015 & 0,500 & 0,500 & \multirow{5}{*}{$\begin{array}{l}\text { ASTM C1609 } \\
\text { modificada }\end{array}$} \\
\hline Primero & 0,085 & 0,325 & 0,825 & \\
\hline Segundo & 0,135 & 0,375 & 1,200 & \\
\hline Tercero & 0,185 & 0,425 & 1,625 & \\
\hline Cuarto & 0,235 & 1,625 & 3,000 & \\
\hline
\end{tabular}




\section{RESULTADOS Y DISCUSIÓN}

\section{Caracterización De Agregados y Cemento}

La Tabla 2 es un resumen de las propiedades obtenidas para el agregado fino y grueso. Adicionalmente, la Figura 3 corresponde a sus curvas granulométricas.
Ambos agregados tienen una gradación continua y además cumplen con la distribución de tamaño tolerada de acuerdo con ASTM C33-18, siendo que la curva experimental se encuentra entre los límites mínimo y máximo. En ambos casos hay cierta tendencia de la distribución a encontrarse más cerca del tamaño más pequeño admisible. El módulo de finura se calculó como 7,05 para el AG y 3,27 para el AF. La gravedad específica del cemento fue de 3,07.

\begin{tabular}{|c|c|c|}
\hline \multicolumn{2}{|c|}{ Tabla 2. Propiedades determinadas para los dos agregados utilizados } \\
\hline \multirow{2}{*}{ Parámetro } & \multicolumn{2}{|c|}{ Agregado } \\
\cline { 2 - 3 } & Grueso & 2,63 \\
\hline $\mathrm{GE}_{\mathrm{SSD}}$ & 2,680 & 2,55 \\
\hline $\mathrm{GE}_{\mathrm{OD}}$ & 2,630 & 1650 \\
\hline $\mathrm{PU}_{\mathrm{OD}-\mathrm{v}}\left(\mathrm{kg} / \mathrm{m}^{3}\right)$ & 1540 & 1700 \\
\hline $\mathrm{PU}_{\mathrm{SSD}-\mathrm{v}}\left(\mathrm{kg} / \mathrm{m}^{3}\right)$ & 1570 & 35,37 \\
\hline $\operatorname{Vacíos}_{\mathrm{OD}-\mathrm{v}}(\%)$ & 41,21 & 5,647 \\
\hline Humedad $(\%)$ & 0,748 & 3,271 \\
\hline Absorción $(\%)$ & 1,858 & 3,27 \\
\hline Módulo de fineza $(\mathrm{MF})$ & 7,050 & \multicolumn{2}{|c|}{} \\
\hline
\end{tabular}

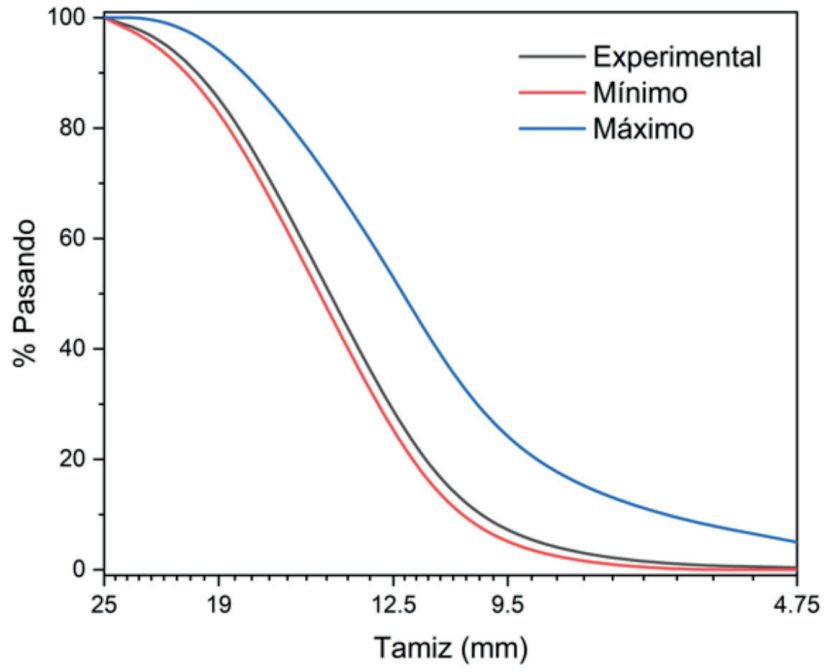

(a)

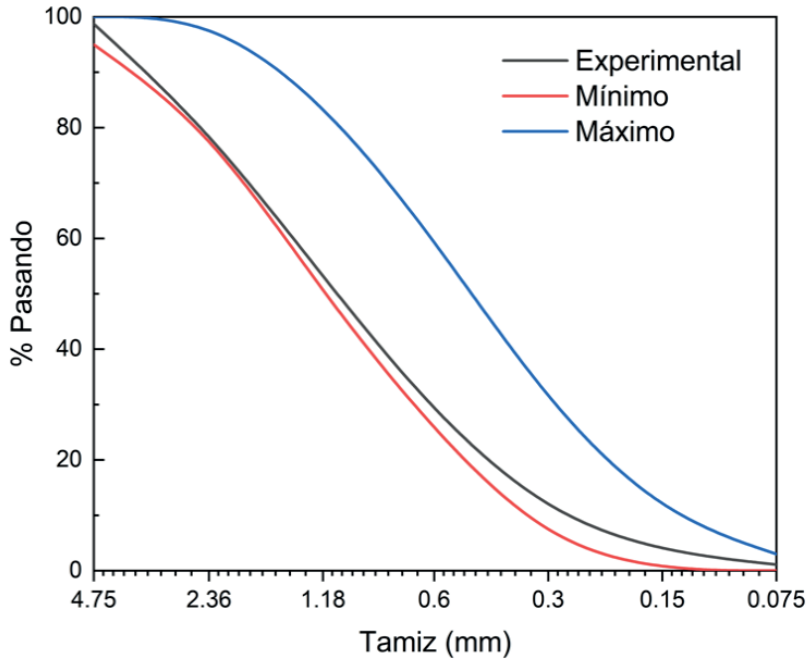

(b)

Figura 3. Curvas granulométricas para a) agregado fino y b) grueso. 


\section{Mezclas de concreto de prueba}

Se prepararon en total tres mezclas de prueba. Para la primera las proporciones fueron establecidas según el procedimiento dado por el Instituto Americano del Concreto en su reporte ACI 211.1-91 (ACI Comittee 211, 1991); para la segunda y tercera mezcla las proporciones fueron ajustadas de acuerdo a las necesidades dadas según los resultados obtenidos para las resistencias a compresión de la Tabla 3, el código de probeta se especifica en la sección de metodología.

\begin{tabular}{|l|c|}
\hline \multicolumn{2}{|c|}{ Tabla 3. Resistencia a compresión } \\
de las mezclas de prueba \\
\hline Probeta & Resistencia a compresión promedio \\
(Mpa)
\end{tabular}

\section{Fabricación De Las Vigas de SNFRC}

Se debe indicar que al momento de iniciar la fabricación de las vigas con baja dosis de fibra no se contaba con los resultados de resistencia a compresión a 28 días para los cilindros correspondientes a la segunda y tercera mezcla de prueba, por ello se utilizaron relaciones teóricas dadas en el reporte ACI 209R-92 (ACI Comittee 209, 1992) con el fin de proyectar la razón W/C requerida para obtener una resistencia cercana a $28 \mathrm{MPa}$ a partir de los valores $\mathrm{W} / \mathrm{C}$ de las mezclas 2 y 3 y la resistencia a los 7 días para tales mezclas. Se obtuvo que era requerido una proporción aproximada $\mathrm{W} / \mathrm{C}=0,79$. $\mathrm{Al}$ iniciar la fabricación de las vigas con baja dosis de fibra la mezcla se observaba demasiado seca y poco trabajable por lo que se realizó un ajuste en la relación $\mathrm{W} / \mathrm{C}$, alcanzando un valor cercano a 0,95 . Las proporciones usadas para fabricar las vigas con baja dosificación de fibra de $1,5 \mathrm{~kg} / \mathrm{m}^{3}$ se muestran en la Tabla 4.

\begin{tabular}{|c|c|}
\begin{tabular}{|c|} 
Tabla 4. $\boldsymbol{f}_{\mathrm{c}}$ de los cilindros fabricados con cada \\
grupo de vigas1.
\end{tabular} \\
\hline $\begin{array}{c}\text { Probeta } \\
\text { CBAS }\end{array}$ & $\begin{array}{c}\text { Resistencia a compresión promedio } \\
\text { (MPA) }\end{array}$ \\
\hline CBASM & 16,6 \\
\hline CBEN & 14,4 \\
\hline CAAS & 17,6 \\
\hline CAASM & 19,4 \\
\hline CAEN & 24,1 \\
\hline
\end{tabular}

De la Tabla 4 se desprende que esta mezcla resultó con baja resistencia pues alcanzó solo 16,6 $\mathrm{MPa}$, el contenido de agua fue demasiado alto. Por ello, para producir las vigas con alta dosis de fibra las proporciones usadas fueron las de la tercera mezcla de prueba, y se dan en la Tabla 5.

\begin{tabular}{|c|c|c|}
\hline \multicolumn{3}{|c|}{ Tabla 5. Proporciones de mezcla para la fabricación de las vigas de SNFRC } \\
\cline { 2 - 3 } Componente & \multicolumn{2}{|c|}{ Cantidad en masa $( \pm 0,01 \mathrm{~kg})$} \\
\hline & Mezcla dosis baja $\left(0,063 \mathrm{~m}^{3}\right)$ & Mezcla dosis alta $\left(0,060 \mathrm{~m}^{3}\right)$ \\
\hline Agregado grueso & 56,12 & 56,79 \\
\hline Agregado fino & 64,48 & 61,08 \\
\hline Cemento & 14,84 & 26,1 \\
\hline Agua & 13,68 & 20,82 \\
\hline W/C & 0,95 & 0,82 \\
\hline Aditivo & $200 \mathrm{ml}$ & -- \\
\hline & \multicolumn{2}{|c|}{ Cantidad en masa $( \pm 0,1 \mathrm{~g})$} \\
\hline Fibra & 94,5 & 330,2 \\
\hline
\end{tabular}


Todas las probetas de la tabla anterior contenían fibra y debe tomarse en cuenta que existe la posibilidad de que el valor obtenido sea en realidad algo menor que el del concreto si estuviese no reforzado. Esto puede deberse a la distorsión generada por las fibras en el concreto, especialmente aquellas orientadas de manera perpendicular a la dirección de aplicación de la carga (Grzymski, Musial, \& Trapko, 2019).

\section{Ensayos de flexión con el SNFRC}

Tanto para los ensayos según ASTM C1609 como por la norma EN14651 se utilizaron puntos de apoyo sin capacidad rotatoria y separados entre sí por una distancia de $\mathrm{L}=450$ mm. Más adelante, en la sección de Ensayos con las vigas de alta dosificación de fibra se presentará un resumen de los parámetros calculados en los ensayos.

\section{Ensayos con las vigas de baja dosificación de fibra $\left(1,5 \mathrm{~kg} / \mathrm{m}^{3}\right)$}

La Figura 4 corresponde a los resultados del ensayo de las vigas con baja dosificación y según ASTM C1609 sin modificaciones.

Las vigas BAS1, BAS3 y BAS4 no evidenciaron ninguna resistencia otorgada por las fibras más allá de su esfuerzo máximo en flexión pues una vez formada la primera grieta el ensayo se detuvo. Aunque se utilizó la velocidad mínima dada por la norma, esta resulta muy alta para ensayar SNFRC con baja dosis de fibra, aun cuando la matriz es un concreto de resistencia baja y que por tanto tiene una fragilidad que, relativamente, debe ser menor (Banthia \& Islam, 2013).

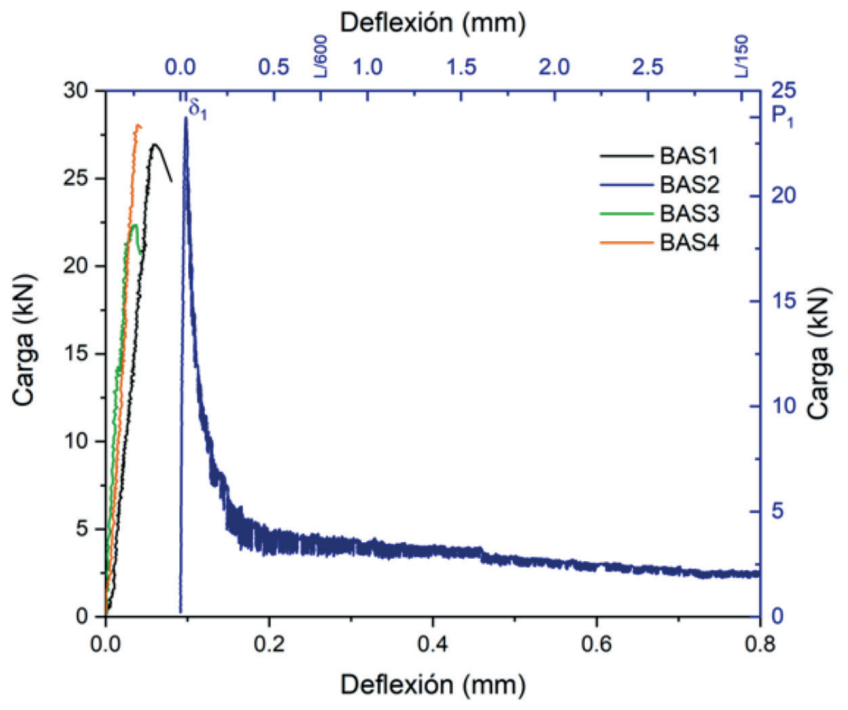

Figura 4. Carga vs Deflexión para vigas con baja dosificación de fibra ensayadas según ASTM C1609 estandar. Los ejes en azul se asocian únicamente con BAS2.
Esta situación se debe a la problemática que presenta la norma americana, se produce una acumulación de energía en el interior del concreto durante la etapa inicial del ensayo; esta energía se libera de manera abrupta (Valenzuela, 2010) y la máquina de ensayo percibe, a través del LVDT, un desplazamiento vertical muy alto súbitamente, y el ensayo se detiene.

El comportamiento en la probeta BAS2 fue distinto ya que sí es clara su capacidad residual siendo que la viga resistió alrededor de unos $5 \mathrm{kN}$ aún después de alcanzado su MOR. Este comportamiento es totalmente aleatorio y puede estar relacionado con condiciones específicas en esa viga en cuanto a la distribución de agregados y fibras así como su orientación (Conforti, Minelli, Plizzari, \& Tiberti, 2018). Con las relaciones dadas en la norma ASTM C1609 se puede determinar la razón de resistencia a flexión equivalente para esta viga: $R_{T, 150}^{D}=16,80 \%$.

El siguiente conjunto de 4 vigas se ensayó de acuerdo con ASTM C1609 pero con la modificación planteada. La velocidad para el primer tramo de deflexión se redujo a aproximadamente la mitad de la velocidad más lenta admitida por la norma, es decir, de $0,035 \mathrm{~mm} / \mathrm{min}$ hasta $0,015 \mathrm{~mm} / \mathrm{min}$ para permitir una acumulación de energía menos agresiva en las vigas. En la Figura 5 se encuentran los resultados de este ensayo.

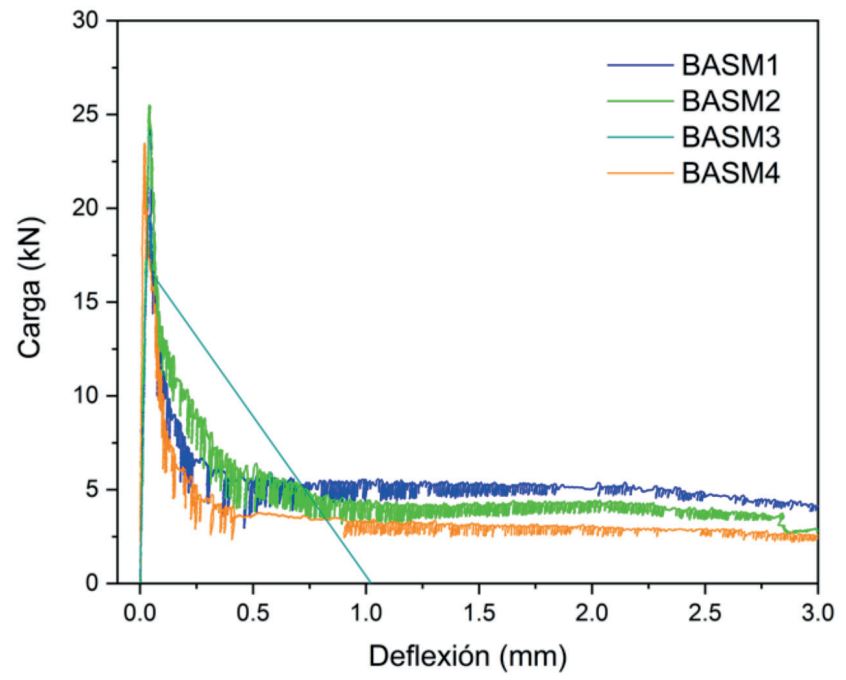

Figura 5. Carga vs Deflexión para vigas con baja dosificación de fibra ensayadas según ASTM C1609 modificada. 
En este caso tres de las vigas atravesaron un ensayo exitoso y sí mostraron resistencia residual. Los resultados indican que con solo variar la velocidad del primer tramo de deflexión (hasta $0,5 \mathrm{~mm}$ ) el ensayo tiene mayor probabilidad de exito, por lo que es en la primera etapa del ensayo en la que se encuentra su periodo crítico. La viga BASM3 experimentó una grieta de manera súbita y por ello el ensayo no alcanzó los $3 \mathrm{~mm}$ de deflexión, esto puede deberse de nuevo a factores aleatorios en la estructura interna de esa viga.

El comportamiento residual de las vigas BASM1 y BASM2 fue muy similar, se obtuvieron valores de $R_{T, 150}^{D}$ de $22,3 \%$ y 19,0 $\%$, respectivamente. La viga BASM4 presentó menores resistencias residuales y con ello su $R_{T, 150}^{D}$ es más bajo, de $15,5 \%$. El fenómeno de reducción y aumento constante de la carga en los gráficos es debido a los pequeños ciclos de carga y descarga que experimentan las vigas gracias a la presencia de las fibras ya que los esfuerzos actúan de manera alternada en la fibra y la matriz.

En la Figura 6 se muestra el comportamiento de las vigas de la serie BENX, es decir, con baja dosis y ensayadas por EN14651. Todos los ensayos fueron exitosos pues la muesca que se hace a las vigas permite una formación de grieta direccionada.

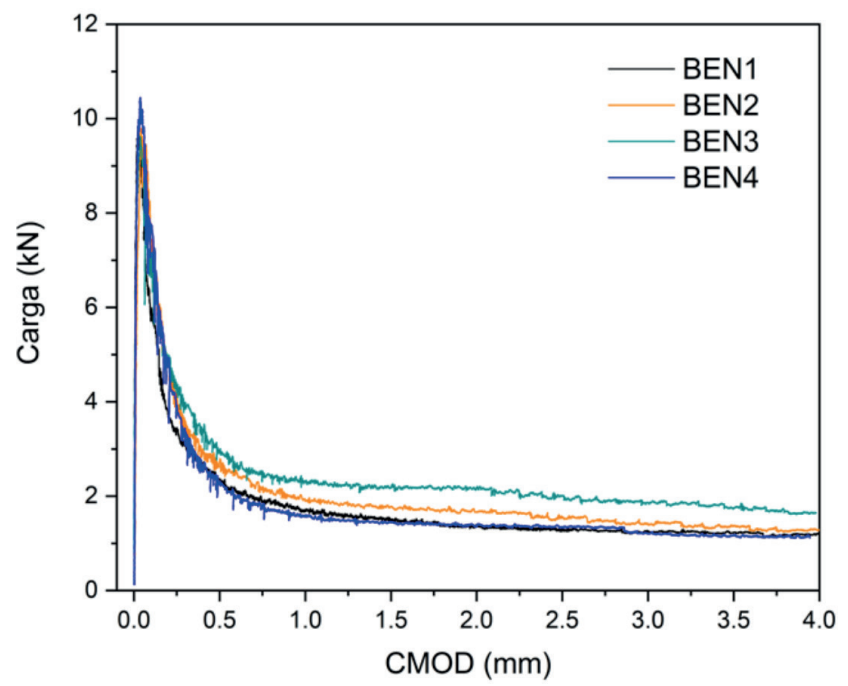

Figura 6. Carga vs CMOD para vigas con baja dosificación de fibra ensayadas por EN14651.

Se determina que las capacidades residuales calculadas según ambas normas son valores cercanos. El valor promedio obtenido para la norma americana modificada fue de $18,93 \%$ y de 20,98\% para la norma europea. Las cargas residuales soportadas por las vigas son ligeramente menores el ensayo con la configuración de EN14651 porque en las vigas del grupo BENX existe una pequeña parte del área transversal en la que no hay ni matriz ni fibras actuando pues está presente la muesca.
Análogamente, ambos ensayos arrojaron valores promedio cercanos para el esfuerzo correspondiente al MOR del concreto, los cálculos de $f_{1}$ y $f_{c t, L}^{f}$ varían solo un $8,20 \%$. De estas observaciones se extrae que los resultados del ensayo vía ASTM C1609 modificada y EN14651 fueron bastante comparables para el caso de baja dosificación de fibra.

\section{Ensayos con las vigas de alta dosificación de fibra $\left(5,5 \mathrm{~kg} / \mathrm{m}^{3}\right)$}

Como se aprecia en la Figura 7, las vigas con dosificación alta de fibra pudieron ser ensayadas sin problemas por el método ASTM C1609 estandar y es evidente que el alto contenido de fibra produce una menor caída en la carga después del MOR que en los casos anteriores.

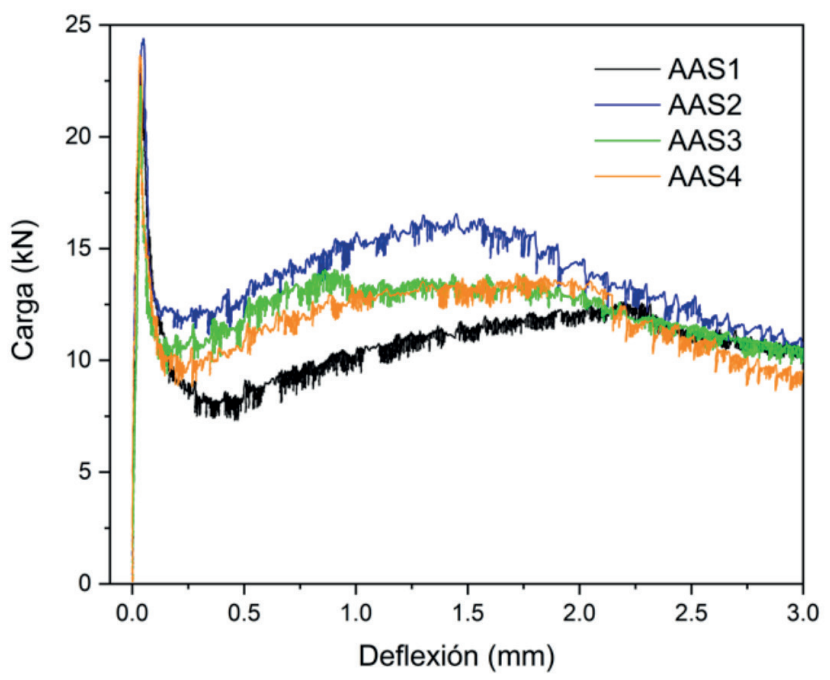

Figura 7. Carga vs Deflexión para vigas con alta dosificación de fibra ensayadas por ASTM C1609 estándar.

Los resultados coinciden con lo reportado por Banthia e Islam, quienes afirman que la norma americana comúnmente arroja curvas inestables para los casos de concreto con baja dosis de fibra, mas no así para cantidades mayores a $3 \mathrm{~kg} / \mathrm{m}^{3}$ (Banthia \& Islam, 2013). Para reforzar este punto de vista, debe recalcarse que el funcionamiento del ensayo es una consecuencia solamente de la incorporación de $5,5 \mathrm{~kg} / \mathrm{m}^{3}$ ya que, de acuerdo con la Tabla 4, el concreto que se utilizó para las vigas del grupo AASX es incluso algo más resistente, y con ello más frágil, que el utilizado para las vigas de la serie BASX.

Al ejecutar el ensayo con las vigas de alta dosificación y la versión modificada de la norma ASTM C1609 se obtuvieron los resultados de la Figura 8. El aumento en la resistencia $f_{c}$ de las vigas de alta dosificación respecto a las de baja (ver Tabla 4) no tuvo gran efecto sobre las cargas pico. Los valores de esfuerzo en esa carga, $f_{1}$, no distan mucho entre sí, siendo la variación promedio entre los grupos BASMX y AASMX de un 4,6\% para ese parámetro. 


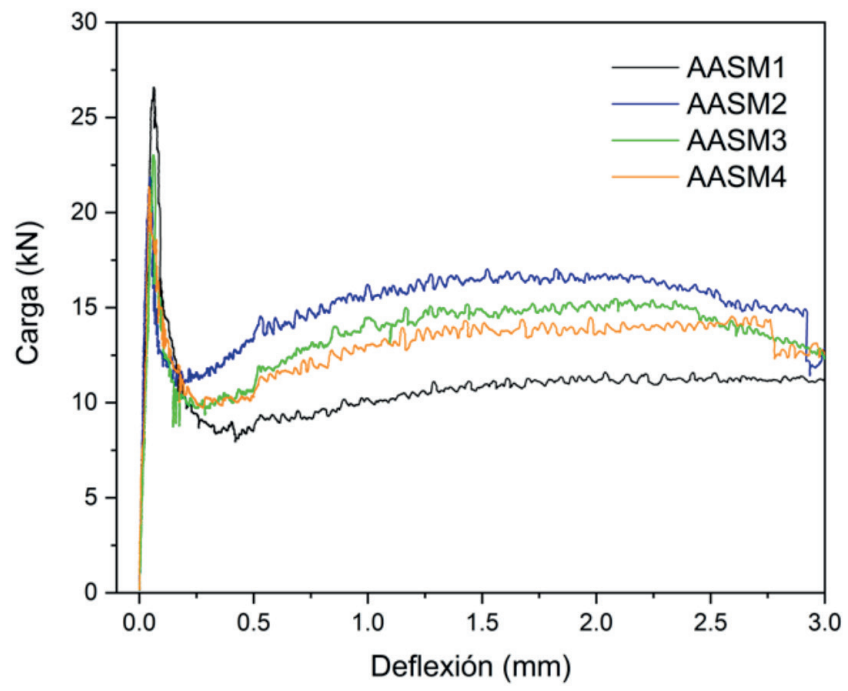

Figura 8. Carga vs Deflexión para vigas con alta dosificación de fibra ensayadas por ASTM C1609 modificada.

Es claro el efecto que tiene la mayor incorporación de fibras sobre el comportamiento residual del SNFRC. Se nota en la Figura 8 que existe un área mucho mayor bajo las curvas que para los gráficos de la Figura 4, lo cual indica mucha más tenacidad en las vigas con dosis de $5,5 \mathrm{~kg} / \mathrm{m}^{3}$.

También es importante señalar que, al comparar los resultados de las Figuras 7 y 8, se nota que no hay una disminución notable en el valor del MOR al utilizar la mitad de la velocidad mínima dada por ASTM C1609 para el primer rango de deflexión, lo cual es una preocupación que existe cuando se disminuyen las tasas de deformación que establece norma (Banthia \& Islam, 2013), en este caso los resultados muestran un MOR que ocurre en general dentro del rango de 20 kN a 25 kN aproximadamente. La modificación no alteró significativamente los resultados.

Para las series AASX y AASXM se obtuvieron valores promedio de $R_{T, 150}^{D}$ de $52,05 \%$ y $57,60 \%$, respectivamente. Estos valores duplican el valor calculado para las vigas de serie BASM (vigas de la Figura 4). De entre todas las vigas es destacable el alto valor de $R_{T, 150}^{D}$ mostrado por la probeta AASM2. Los valores individuales de la capacidad residual varían hasta en 30 puntos porcentuales, entre AASM1 y AASM2.

Finalmente se muestran en la Figura 9 los resultados del ensayo con vigas del grupo AENX. El valor del esfuerzo promedio en el MOR, es $f_{c t, L}^{f}=3,21 \mathrm{MPa}$ y se encuentra bastante cercano a los 2,94 MPa y 2,91 MPa que en promedio se determinaron en los casos de los dos ensayos anteriores con ASTM C1609.
En este caso el valor promedio de $\mathrm{R}_{e, 3}$ difiere en un $27 \%$ y $34 \%$ de los promedios de $R_{T, 150}^{D}$ calculados según resultados de las Figuras 7 y 8 . Por ello, para las vigas con más fibra no se cumplió la buena correspondencia que se obtuvo para las capacidades residuales de las probetas con baja dosificación.

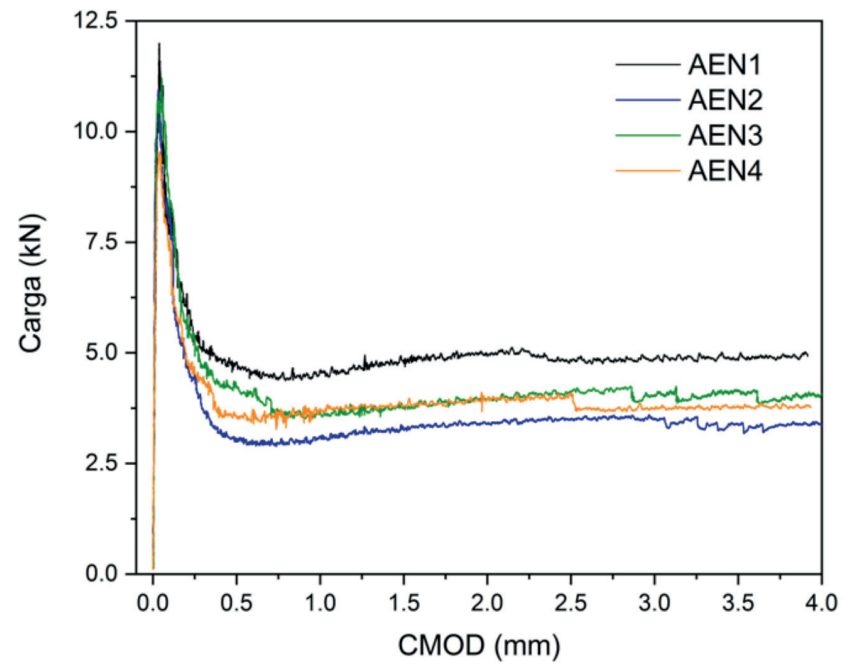

Figura 9. Carga vs CMOD para vigas con alta dosificación de fibra ensayadas por EN14651.

En todos los ensayos se observó el reblandecimiento caracterizado por la caída rápida de la carga después de alcanzado el esfuerzo del primer pico y seguida por un aumento en la resistencia residual, en este tipo de materiales también es característica la formación de una sola grieta que se expande. El fenómeno de reblandecimiento está presente cuando no existe una carga pico que sea mayor a la carga del primer pico en los gráficos (Conforti et al., 2018; Li, Niu, Wan, Liu, \& Jin, 2017). En los gráficos obtenidos por método ASTM el comportamiento residual presenta un máximo cerca de los 1,5 mm de deflexión y luego cae al final del ensayo, por otro lado, para EN14651 la resistencia residual es constante en todo el rango de análisis o bien tiene una ligera tendencia al aumento. Los valores de tenacidad dados en las Tablas 6 y 7 indican que el SNFRC se comporta como un material pseudodúctil que no presenta la fragilidad tradicional del concreto (Nanni, 1991).

Las capacidades residuales $\left(\mathrm{R}_{\mathrm{e}, 3}\right)$ se calcularon según el reporte TR63 (The Concrete Society, 2007) y están dadas en la Tabla 7. 
Tabla 6. Resultados para vigas ensayadas según ASTM C 1609 corriente y modificada.

\begin{tabular}{|c|c|c|c|c|}
\hline \multirow{2}{*}{ Código de viga } & \multicolumn{4}{|c|}{ Parámetro } \\
\hline & $P_{1}(k N)$ & $f_{1}(M p a)$ & $T_{150}^{D}(J)$ & $R_{T, 150}^{D}(\%)$ \\
\hline \multicolumn{5}{|c|}{ SERIE BASMX } \\
\hline BASM1 & 24,3 & 3,05 & 16,0 & 22,3 \\
\hline BASM2 & 25,5 & 3,15 & 15,0 & 19,0 \\
\hline BASM3 & 19,6 & 2,45 & - & - \\
\hline BASM4 & 23,5 & 2,95 & 11,0 & 15,5 \\
\hline Promedio & 24,4 & 3,05 & 14,0 & 18,9 \\
\hline Desv. Estándar & 0,83 & 0,08 & 2,16 & 2,78 \\
\hline \multicolumn{5}{|c|}{ SERIE AASX } \\
\hline AAS1 & 23,2 & 2,93 & 32,0 & 46,6 \\
\hline AAS2 & 24,4 & 3,03 & 41,0 & 56,6 \\
\hline AAS3 & 22,3 & 2,81 & 37,0 & 54,9 \\
\hline AAS4 & 23,7 & 2,98 & 36,0 & 50,1 \\
\hline Promedio & 23,4 & 2,94 & 36,5 & 52,1 \\
\hline Desv. Estándar & 0,77 & 0,08 & 3,2 & 3,95 \\
\hline \multicolumn{5}{|c|}{ SERIE AASMX } \\
\hline AASM1 & 26,6 & 3,34 & 32,0 & 40,7 \\
\hline AASM2 & 21,9 & 2,73 & 45,0 & 69,4 \\
\hline AASM3 & 23,0 & 2,89 & 41,0 & 59,2 \\
\hline AASM4 & 21,4 & 2,68 & 39,0 & 61,1 \\
\hline Promedio & 23,2 & 2,91 & 29,3 & 57,6 \\
\hline Desv. Estándar & 2,04 & 0,26 & 4,70 & 10,5 \\
\hline
\end{tabular}

\begin{tabular}{|c|c|c|c|c|}
\hline \multirow{3}{*}{ Código de viga } & \multicolumn{3}{|c|}{ EN14651. } & \\
\hline & \multicolumn{4}{|c|}{ Parámetro } \\
\hline & $C M O D_{1}(\mathrm{~mm})$ & $f_{c t, L}^{f}(M p a)$ & $D_{3}(J)$ & $R_{e, 3}(\%)$ \\
\hline \multicolumn{5}{|c|}{ SERIE BENX } \\
\hline BEN1 & 0,023 & 2,75 & 5,490 & 18,70 \\
\hline BEN2 & 0,042 & 2,78 & 6,340 & 21,60 \\
\hline BEN3 & 0,034 & 2,76 & 7,460 & 25,80 \\
\hline BEN4 & 0,037 & 2,91 & 5,590 & 17,80 \\
\hline Promedio & 0,034 & 2,80 & 6,220 & 20,98 \\
\hline Desv. Estándar & 0,007 & 0,06 & 0,79 & 3,12 \\
\hline \multicolumn{5}{|c|}{ SERIE AENX } \\
\hline AAS1 & 0,038 & 3,46 & 14,8 & 41,1 \\
\hline AAS2 & 0,033 & 3,26 & 10,6 & 32,1 \\
\hline AAS3 & 0,049 & 3,30 & 12,5 & 37,5 \\
\hline AAS4 & 0,040 & 2,80 & 11,8 & 41,2 \\
\hline Promedio & 0,040 & 3,21 & 12,4 & 38,0 \\
\hline Desv. Estándar & 0,006 & 0,250 & 1,53 & 3,70 \\
\hline
\end{tabular}

\section{CONCLUSIONES}

Se evidenció el comportamiento de reblandecimiento del SNFRC; el de alta cantidad de fibra demostró ser más tenaz y resistente a cargas residuales. La variación en la resistencia a compresión de las distintas coladas no generó diferencias importantes en los gráficos de los distintos ensayos; incluso, el módulo de ruptura, MOR, del concreto con poca fibra resultó muy cercano al de alta fibra, aun cuando las proporciones de mezcla fueron distintas. La dispersión de los resultados de cada ensayo individual se identificó como una característica aleatoria.

La norma ASTM C1609 no permite estudiar, de manera confiable, el SNFRC con baja dosificación de fibra, sin embargo, un ligero cambio en la velocidad estipulada da pie a mayor probabilidad de éxito en el ensayo. La norma no presenta problemas cuando hay alta cantidad de fibra en el concreto. Además, la modificación de la velocidad no alteró los resultados ya que los valores promedio arrojados para capacidad residual y resistencia en el primer pico variaron solamente $9,6 \%$ y $1,0 \%$, respectivamente y para el caso con $5,5 \mathrm{~kg} / \mathrm{m}^{3}$

El estándar EN14651 permite que el ensayo de flexión se ejecute sin problemas, pero la capacidad residual obtenida solo es comparable con lo dado por ASTM C1609 para el caso con baja dosificación pues la variación promedio entre las capacidades residuales y fue de 9,8\% mientras para el SNFRC con $5,5 \mathrm{~kg} / \mathrm{m}^{3}$ de fibra la diferencia alcanzó hasta $34,1 \%$. El aceptar o no la diferencia entre normas es una apreciación subjetiva del interesado en los ensayos.

El ensayo por EN14651 requiere mayor tiempo para preparar las vigas al generarles la muesca y para colocar las placas para medición de su apertura, no considera el efecto de cualquier microgrieta formada porque el sensor únicamente mide la apertura de la muesca. La carga y tenacidad que el ensayo arroja son inferiores a los reales debido a la alteración de la sección y esto hace que para casos en los que se requieren estudios rápidos este ensayo no sea tan conveniente porque requiere el tratamiento de los datos para obtener valores calculados de MOR y deflexión. La norma americana, en cambio, provee resultados más fáciles de interpretar porque mide deflexión de manera directa y la tenacidad es la verdadera presentada. 


\section{AGRADECIMIENTOS}

Al personal profesional y técnico del LanammeUCR por su soporte para la ejecución de los varios ensayos. Finalmente, Ing. Oscar Chaverri Quirós M.Sc de Tecnológico de Costa Rica por sus aportes en el desarrollo de la investigación.

\section{REFERENCIAS}

ACI Comittee 209. (1992). ACI 209: Prediction of Creep, Shrinkage, and Temperature Effects in Concrete Structures (Reapproved 1997). ACI Structural Journal.

- ACI Comittee 211. (1991). ACI 211.1-91: Standard Practice for Selecting Proportions for Normal, Heavyweight, and Mass Concrete (Reapproved 2009). ACI Structural Journal.

ACI Comittee 544. (1996). ACI 544-1R: State-of-the-Art Report on Fiber Reinforced Concrete (Reapproved 2002). ACI Structural Journal.

- ASTM International. (2009). ASTM C1116/C1116M-10a: Standard Specification for Fiber-Reinforced Concrete. Annual Book of ASTM Standards. West Conshohocken, PA. http://doi. org/10.1520/C1116

ASTM International. (2012). ASTM C1609/C1609M-12: Standard Test Method for Flexural Performance of Fiber-Reinforced Concrete (Using Beam With Third-Point Loading). Annual Book of ASTM Standards. West Conshohocken, PA.

Banthia, N., \& Islam, S. T. (2013). Loading Rate Concerns in ASTM C1609. Journal of Testing and Evaluation, 41(6), 20120192. http://doi.org/10.1520/jte20120192

Conforti, A., Minelli, F., Plizzari, G. A., \& Tiberti, G. (2018). Comparing test methods for the mechanical characterization of fiber reinforced concrete. Structural Concrete, 19(3), 656-669. http://doi.org/10.1002/suco.201700057

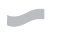

European Comittee for Standardization. (2005). EN 14651: Test Method for Metallic Fibered Reinforced Concrete-Measuring the Flexural Tensile Strength (Limit of Proportionality (LOP), residual). Rue de Stassart, Brussels.

Grzymski, F., Musial, M., \& Trapko, T. (2019). Mechanical Properties of Fibre Reinforced Concrete With Recycled Fibre. Construction and Building Materials, 198, 323-331.

Kosmatka, S. H., Kerkhoff, B., \& Panaresse, W. C. (2002). Design and Control of Concrete Mixtures (14th ed.). Skokie, Illinois: Portland Cement Association (PCA).
Li, J., Niu, J., Wan, C., Liu, X., \& Jin, Z. (2017). Comparison of flexural property between high performance polypropylene fiber reinforced lightweight aggregate concrete and steel fiber reinforced lightweight aggregate concrete. Construction and Building Materials, 157, 729-736. http://doi.org/10.1016/j.conbuildmat.2017.09.149

Nanni, A. (1991). Pseudoductility of Fiber Reinforced Concrete. Journal of Materials in Civil Engineering, 3(1), 78-90. http:// doi.org/10.1061/(asce)0899-1561(1991)3:1(78)

Navas Carro, A., \& Rojas Juárez, J. L. (2010). Comportamiento de losas apoyadas en suelo utilizando concreto reforzado con fibras metálicas. Ingeniería, 20(1 y 2), 67-80.

P. Kumar Mehta, P. D., \& Paulo J. M. Monteiro, P. D. (2014). Special Types of Concrete. In Concrete: Microstructure, Properties, and Materials, Fourth Edition. McGraw Hill Professional, Access Engineering. Retrieved from https://www.accessengineeringlibrary.com:443/browse/concrete-microstructure-properties-and-materials-fourth-edition/c9780071797870ch12

Paegle, I., \& Fischer, G. (2014). Evaluation of test methods used to characterize fiber reinforced cementitious composites. Proceedings of the International Conference "Innovative Materials, Structures and Technologies," 122-128.

The Concrete Society. (2007). Technical Report No. 63: Guidance for the Design of Steel-Fibre-Reinforced Concrete. UK.

Valenzuela, M. (2010). Estudio del comportamiento de hormigones con fibras estructurales en pavimentos. Universidad de Los Andes.

Zongjin, L. (2017). Advanced Concrete Technology. Advanced Concrete Technology. Hoboken, New Jersey: John Wiley \& Sons. 